\title{
Molecular Dynamic Approach to Predict the Miscibility of Excipients for Lipid-based Formulations.
}

\author{
Murugesan Anand Babu
}

PSG College of Pharmacy

Radhakrishnan Nithya ( $\mathbf{8 2 n i t h i k r i s h @ g m a i l . c o m ~ ) ~}$

PSG College of Pharmacy https://orcid.org/0000-0003-1518-4116

\section{Veintramuthu Sankar}

PSG College of Pharmacy

\section{Nithyananth Muniswamy}

PSG College of Pharmacy

\section{Research Article}

Keywords: Solubility parameter, Molecular dynamics, Miscibility.

Posted Date: March 7th, 2022

DOI: https://doi.org/10.21203/rs.3.rs-1287799/v1

License: (c) (i) This work is licensed under a Creative Commons Attribution 4.0 International License. Read Full License 


\section{Abstract}

This research work sought to investigate the miscibility of various excipients with Nebivolol hydrochloride (NBV) to develop stable lipid-based nanoparticles. The miscibility of NBV with excipients such as Glyceryl Mono Oleate (GMO), VIT-E TPGS and Poloxamer 407 was predicted using molecular simulation. The miscibility predicted by in-silico approach was corroborated with a thermogram obtained from Differential Scanning Calorimetry (DSC). Desmond MD engine using OPLS-3e force field was used to perform molecular dynamic (MD) simulations. The cohesive energy density and the solubility parameters $(\delta)$ were determined for all the compounds. Solubility parameters computed from the simulation indicate that the mixture of NBV/GMO and NBV/VITE-TPGS are miscible with each other. The miscibility is determined by the difference in solubility parameter between drug and carrier. DSC thermogram observations have substantiated the miscibility of NBV /GMO, NBV/Poloxamer 407 and NBV/VITE-TPGS. This finding indicates that Nebivolol hydrochloride is soluble in GMO and this combination is further miscible with Poloxamer 407. NBV/POLOXAMER 407 is predicted as immiscible. With this study, we report that the molecular dynamic simulation can potentially be used to understand the molecular level insights to develop the thermodynamically stable formulation.

\section{Introduction}

Nowadays the drug discovery process results in an increasing number of drug candidates with very substandard pharmaceutical properties. Nearly $75 \%$ of APIs have poor aqueous solubility and least physical stability. This may lead to hindrance to drug dissolution in the Gl tract which invariably affects the bioavailability of oral dosage form [1]. This has been overcome by enhancing the API solubility with the use of drug delivery technologies such as particle amorphization, lipid-based systems, size reduction, salt formation, self-emulsification, complexation with cyclodextrins, cosolvent systems, micellar and surfactant systems [2].

Most of the formulations mentioned above are prepared using various excipients such as lipids and polymers. Several physical properties of the excipients which influence the formulation have been identified in pre-formulation studies. The formulation characteristics such as stability, drug loading, and retention are directly influenced by the solubility/miscibility of the drug in the excipient and specific interactions between them. The miscibility of the drug with excipient serves as an indicator to formulate the stable dosage forms [3].

Solubility parameters of formulation components are vital for the selection of excipients with desired properties to achieve expected performance. First, an excipient must have specific bonding interaction with API via weak van der Waals interactions or by hydrogen bonding. The carrier must overcome intermolecular forces that bind the drug together. In some cases, the drug may not be miscible with the carrier; rather it swells or softens completely. If the swelling is uniform, the drug molecules will be able to place themselves between the carrier molecules and weaken the intermolecular forces thereby enhancing the solubility. Solubility parameters are excellent solubility predictors for the non-hydrophilic polymeric 
carrier with no hydrogen bonds. By using this, solubility enhancement of poorly soluble drugs such as Lacidipine, Indomethacin, Itraconazole, Lamivudine, Zidovudine, Ibuprofen, Lafutidine, Carbamazepine, Propranolol $\mathrm{HCl}$, and Diphenhydramine $\mathrm{HCl}$ have been achieved [4, 5]. The insight about phase behavior of drug-polymer forewarns the phase separation brought up by supersaturation of drug in a particular polymer. To study the science involved in this phase behavior, computational methods can be used to predict the atomic-level interactions of components in a system. By using such molecular level insights obtained from in-silico modeling studies, the process behind the solubility enhancement is expediated during the formulation development [6].

In-silico tools such as molecular dynamic simulations have been developed to aid the solubility improvement of drugs and can be combined along with in-vitro studies [7]. MD is based on the fundamental physics of a forcefield that combines the intermolecular and intramolecular forces experienced by the molecules and gives a perspective of the system's dynamic evolution. Various methods based on $\mathrm{H}$-bonding interaction, Van der Waal forces, covalently linked atoms, and electrostatic forces between the molecules in a system have been devised. The kinetic motion of molecules is affected by forces experienced between the molecules and as a result, the location of the molecule changes over time. In most cases, the algorithm computes per atom and then integrated it for the entire system [8]. Hence understanding the combination of molecular dynamics with current solubilization technology is essential for in-silico predictions [9].

Solubility parameter based component selection is established through the cohesive energy of the components, which is the net consequence of all interatomic/molecular interactions. The solubility of excipients, polymers, drugs, and oils is calculated using the Hildebrand and Hansen solubility parameters [10]. Hildebrand SP is based on the heat of vaporization which can be applied for compounds with polar and hydrogen bonds. Hansen SP is based on cohesive energy contributed by various forces such as dispersion forces, hydrogen bonds, and dipolar interactions $[8,11,12]$.

Hansen SP $\delta=\sqrt{\frac{\Delta h v-R T}{v_{m}}}$

$$
\text { Hildebrand } \mathrm{SP} \delta_{s}^{2}=\delta_{d}^{2}+\delta_{p}^{2}+\delta_{h}^{2}
$$

Recent increase in publications and patents in lipid-based delivery has been a reason to develop various computational models to predict the miscibility between the drug and polymer or polymer blends of these components in the lipid mixture [13-15]. The advantage of lipid-based formulation is that the drug is dissolved within the lipid-polymer mixture, therefore, presenting the drug in a pre-solubilised form in the GIT. So the solubility in the lipid-polymer mixture is important to achieve biopharmaceutical advantages. Hence, prediction and ranking of drug solubility in a wide range of lipids, polymer mixtures may use to aid development of lipid-based formulation $[10,16,17,18]$. However, these experiments are costly, timeconsuming, resource-intensive and the experimental protocols vary substantially between different 
research groups [19]. The findings obtained from PubMed from 1996 to 2016 indicate only $9 \%$ of lipidbased drug products have been commercialized [20]. The development of new sophisticated in-silico simulation and modeling techniques facilitates the prediction of drug solubility in lipids. In which molecular structure, physicochemical properties, and particular solute-solvent interactions may be taken into consideration to predict the drug solubility in lipids to improve the drug loading and encapsulation properties for the preparation of lipid-based formulations such as solid lipid nanocarrier, nanostructured lipid carrier, cubic phases, micellar solutions and self-emulsifying drug delivery system (SEDDS).

The goal of this study is to determine the miscibility of Nebivolol hydrochloride in different excipients such glycerol mono oleate as lipid and poloxamer 407, Vit E TPGS as polymer. The above objective was achieved with the help of solubility parameter computed from molecular dynamic simulations and the results were corroborated with thermal analytical techniques.

\section{Materials}

Nebivolol HCL (gift sample), Glycerol mono oleate (GMO) (Mohini Organics Pvt Ltd, Mumbai, India), Pluronic F127 (Fisher scientific India Ltd., Mumbai) and Vit-E TPGS (Sigma Aldrich, Bangalore, India).

\section{Methods}

\subsection{Determination of solubility parameter using Molecular Dynamic Simulation}

The MD simulations were performed using SCHRÖDINGER MATERIALS SCIENCE SUITE Version 21.x. Molecular structure of Nebivolol HCL, Glycerol mono oleate and VIT-E TPGS were obtained from PubChem canonical smile strings (Fig. 1). Each monomer of Pluronic F127, which is an amphiphilic triblock copolymer of PEG100-PPG65-PEG100 (polyethylene glycol 100 repeating units-polypropylene glycol 65 repeating units-polyethylene glycol 100 repeating units), was constructed using a polymer builder panel. The OPLS-3e (optimised potential for liquid simulation) force field was utilised for MD simulations [21].

[Fig. 1 near here.]

\subsection{Disorder System building}

A disordered system was generated for Nebivolol HCl (Molecules), GMO (molecules), VIT-E TPGS (molecules), Pluronic F127 (MW, chains) using disordered system builder. For Pluronic F127 each monomer was sketched by specifying the chemical groups to build the polymer initiator, cascade and terminator after sketching the monomer the amorphous cell tab is used to create a specified number of random polymer containing amorphous cubic cells. The dimensions of amorphous cells are $3 \mathrm{~nm} \times 3 \mathrm{~nm} \times$ $3 \mathrm{~nm}$ with periodic boundary conditions. 
There are various types of protocols available in MD simulation to relax the model before starting the simulations. Materials relaxation is a robust protocol for materials science applications. This consists of a 20 ps NVT Brownian minimisation at $10 \mathrm{~K}$, a 20 ps NPT Brownian minimisation at $100 \mathrm{~K}$ and a 100 ps NPT MD stage at $100 \mathrm{~K}$.

\subsection{Energy Minimization}

Energies of amorphous cubic cells were minimised to avoid unfavorable interactions and to maintain the lowest energy state. The process was done through series of minimisations through material relaxation protocol. The MD simulations were done in two stages: equilibrium and productive phase, normally this option is needed only for model systems that have just been prepared (for example with the System Builder panel) and have not been relaxed. The amorphous cubic cells were permitted to optimise their density in the equilibrium phase using materials relaxation for $10 \mathrm{~ns}$ under isothermal (NPT) or isobaricisothermal conditions (NVT) at 298K. In the production phase, the equilibrated system was allowed to run via multistage workflow panel at $100 \mathrm{~ns}$ for obtaining trajectory frames. Subsequently, trajectory analysis was carried out to calculate the cohesive energy density and solubility parameters of the simulation box.

\section{Theoretical Solubility Parameter}

The theoretical solubility parameter of the components is estimated from the chemical structures using group contribution methods such as Hoftyzer/Van Krevelen and Hoy methods [22].

\section{Differential Scanning Calorimeter (Dsc)}

DSC experiments were performed on DSC3, Mettler-Toledo, Germany). Physical mixtures of Nebivolol $\mathrm{HCl}$ and excipients were prepared in different ratios. During the experiments, aluminum pan containing samples $(7-10 \mathrm{mg})$ were crimped with lids having pinholes. Dry nitrogen is allowed to pass through the DSC chamber at the flow rate equal to $5 \mathrm{ml} / \mathrm{min}$. The melting onset and miscibility of Nebivolol with various excipients are determined by running the samples through a heat-cool-heat cycle. At a rate of $10^{\circ} \mathrm{C} / \mathrm{min}$, samples are heated from $5^{\circ} \mathrm{C}$ to the desired temperature (above or below the melting point).

\section{Results And Discussion}

Energy in each compound is stored in the bonds which can hold indivisible units of energy, known as quanta. There are numerous ways the energy can be distributed, but the total energy of the system always remains the same. This is called microstates. In reality, energy in the system continuously moves between the neighboring bonds. In Molecular Dynamics (MD) simulation, microstates of the system or the distribution of energy between the bonds for the specific set of atoms that interacts are calculated by solving the Newton second law ( $F=m a)$. Numerically, this equation is expressed as a Hamiltonian function which is equal to the sum of the system kinetic and potential energy. The position and velocities 
of an atom in a structure at a later stage were computed from the initial position and velocities by applying the numerical algorithm.

The sum of the valence interactions (E-valence), non-bonding interactions (E non-bond), and the crosscoupling term (E-cross term) in the OPLS3e force- field represents the overall energy (E-total) of a system.

Total $=\mathrm{E}-$ valence $+\mathrm{E}-$ cross- term $+\mathrm{E}$ non - bond (3)

The total potential energy $E$ pot is defined as the sum of many components coming from the parametrization of bonding and nonbonding interactions in this force field, and is represented as follows:

$\mathrm{E}$ pot $=\mathrm{Eb}+\mathrm{E} \theta+\mathrm{E} \phi+\mathrm{E} \chi+\mathrm{E}$ cross $+\mathrm{E}$ Cou $+\mathrm{E} v \mathrm{dW}(4)$

$E b=$ bond stretching energy, $E=$ valence angle bending energy, $E=$ dihedral torsion energy, $E$ = out-of-plane energy, $E$ cross=cross-term interaction energy, $E$ Cou=Coulombic interaction energy, and $E \mathrm{vdW}=\mathrm{van}$ der Waals interaction energy, where $E \mathrm{~b}=$ bond stretching energy, $\mathrm{E}=$ valence angle bending energy, $\mathrm{E}=$ dihedral torsion energy, $E$ = out-of-plane energy, $E$ cross the first five phrases define the short-range bound interactions, whereas the final two define the nonbonded interactions between and inside molecules.

Periodic boundary conditions were used to create amorphous cubic cells for nebivolol hydrochloride, GMO, Poloxamer407, VIT-E TPGS. Energy minimization was imposed on amorphous cubic cells to relax the material, allowing for efficient molecule packing within the amorphous cubic. The effectiveness of the forcefield was assessed by the packing density of the system. Density minimised cells are shown in Fig. 2.

[Fig. 2 near here.]

The time it would take for a disordered system to equilibrate is determined by the number of atoms in it. Density minimised amorphous cubic cells from equilibration phase were used for final MD simulations of the production phase. Table 1 shows the density values obtained in the MD final trajectory analysis. The density values in MD indicate that intermolecular interaction and packing are effective. It indicates that amorphous cell build imitates the original structure. 
Table 1

Density of components retrieved from MD simulations.

\begin{tabular}{|lll|}
\hline SI.NO & COMPOUNDS & DESNITY $\left(\mathbf{g} / \mathbf{c m}^{3}\right)$ \\
\hline 1. & Nebivolol HCL & 1.25 \\
\hline 2. & GMO & 0.95 \\
\hline 3. & Poloxamer 407 & 1.08 \\
\hline 4. & Vit-E TPGS & 1.01 \\
\hline
\end{tabular}

Table 2

solubility Parameters from Group Contribution techniques and MD simulations ( $\mathrm{MPa} 0.5)$

\begin{tabular}{|c|c|c|c|}
\hline compounds & 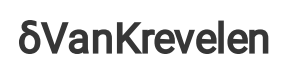 & $\delta$ Hoy & $\delta M D$ simulations \\
\hline Nebivolol HCL & 21.17 & 21.77 & 21.88 \\
\hline GMO & 20.49 & 20.64 & 20.56 \\
\hline Vit-E TPGS & 17.99 & 26.76 & 18.67 \\
\hline $\begin{array}{l}\text { Poloxamer } \\
407\end{array}$ & 11.87 & 13.45 & 14.39 \\
\hline
\end{tabular}

In the production phase, each disorder system was subjected to NPT, MD simulation was set to run for $100 \mathrm{~ns}$ to determine the solubility parameter $\delta$, density, cohesive energy density. The simulations were carried out until the total energy of each system was stabilised. The time, pressure, temperature, potential energy evolution profiles during MD simulations are shown in Fig. 3.

[Fig. 3 near here.]

A quantitative measure of the energy supplied to separate its constituents into neutral free atoms in a molecule is the cohesive energy. It depends on the interaction between polar groups and $\mathrm{H}$-bonding. The cohesive energy per unit of volume is called cohesive energy density. The solubility parameter of the polymer is always defined as the square root of the cohesive energy density in the amorphous state at room temperature. It is widely used for correlating polymer solvent interactions. As a refinement, three solubility parameter components can be distinguished, representing dispersion, polar, and hydrogen bond interactions. As a general rule structural similarity favors solubility besides the chemical structure physical state of a polymer is important for its solubility. Crystalline polymers are relatively insoluble and often dissolve only at temperature slightly below their crystalline melting point. Cohesive energy simulated from MD simulations for VIT -E TPGS is $46.07 \mathrm{Kcal} / \mathrm{mol}$ and it was shown in Fig. 4.

[Fig. 4 near here.] 
Table 1 illustrates the solubility parameter values for nebivolol $\mathrm{HCL}$ and excipients exported from the production stage at $298 \mathrm{~K}$. The values computed from MD simulations are matched up with solubility parameters calculated from theoretical methods such as Hoftyzer-Van Krevelen and Hoy method

As per the Hoftyzer-Van Krevelen method,

$\delta h=\frac{\sum \mathrm{Eh}_{\mathrm{i}}}{\vartheta} ; \delta p=\frac{\sum \mathrm{Ep}_{\mathrm{i}}}{\vartheta} ; \delta d=\frac{\sum \mathrm{Fd}_{\mathrm{i}}}{\vartheta}(5)$

$\boldsymbol{\delta}^{T}=\boldsymbol{\delta}^{2}{ }_{\mathrm{d}} \boldsymbol{\delta}_{\mathrm{p}}^{2}+\boldsymbol{\delta}^{2}{ }_{\mathrm{h}}(6)$

$\mathrm{Eh}_{\mathrm{i}}$ is hydrogen bonding energy, while $\mathrm{V}$ is the molar volume. $\mathrm{Fd}_{\mathrm{i}}$ and $\mathrm{Fp}_{\mathrm{i}}$ are the molar attraction constants attributed to dispersion and polar components respectively. The parameter $\delta$ t stands for total solubility (also referred to as the Hansen solubility parameter). Dispersive, electrostatic-polar, and hydrogen bonding forces are represented by $d, p$, and $h$ respectively.

According to the Hoy approach, the solubility parameter is provided by the following equation.

$\delta^{T}=\frac{\mathrm{F}_{\mathrm{t}}+\mathrm{B}}{\vartheta}$

Where, Ft denotes the molar attraction constant, B denotes the base constant, and $\mathrm{V}$ denotes the molar volume. The density and molecular weight of the substances were used to compute their molar volume. Fdi, Fpi, Ehi, Ft, and B values for different groups were determined using data from the literature.

As shown in Table.2, for each molecule the overall solubility parameter values generated from MD simulations and determined from group contribution approach accord well. This is especially true for NBV and GMO for which the two approaches produced comparable results. In contrast, the solubility parameter values derived from the MD simulations for Vit E TPGS and Poloxamer 407 were moderately lower than those calculated from the theoretical techniques. The dissimilarity can be attributed to the dominance of hydrogen bonding between the molecules of poloxamer 407 and vit E TPGS which cannot be fully characterised by group contribution techniques. From the final MD trajectory, an $\mathrm{H}$-bond interaction graph for NBV and Vit ETPGS was used to depict the H-bond network. As seen in H-bonding graph Fig. 5, Vit E TPGS has a significantly larger H-bond network than Nebivolol HCL.

[Fig. 5 near here.]

The advantage of MD is that it accounts for such directional-dependent interactions during calculations. Group contribution approaches, on the other hand, often do not account for directional interactions, resulting in erroneous CED. The study investigates whether MD simulations can be utilised as a useful screening method and predicts the solubility characteristics of medicinal drugs of interest. Furthermore, computer models can provide insight into the nature of interactions within complex systems at the molecular level. 


\subsection{In-silico prediction of miscibility of drug carrier}

Solubility or miscibility between the compounds may be promoted if both substances contain polar groups or a hydrogen atom. Due to the negative entropy factor, two substances with equivalent solubility parameters are mutually soluble/ miscible. This is in line with the general norm that solubility is favored by chemical and structural similarity. The tendency toward dissolving diminishes as the dissimilarity between two solubility parameters rises. According to the literature, a categorization system based on solubility parameter differences $(\Delta \delta)$ in the molten state has been reported for predicting drug and excipient miscibility and the Compounds with identical values of $\delta$ are thermodynamically miscible. For example, using indomethacin as a model medicine, polypropylene oxide as a polymer, and sucrose and glucose as non-polymeric carriers, Gupta et al. [23] reported that molecules with a less than $7.0 \mathrm{MPa} 0.5$ are likely to be miscible, whereas more than $10.0 \mathrm{MPa} 0.5$ implies an immiscible system.

Table 3

Miscibility of nebivolol HCL with lipid and other excipients from MD Simulations

\begin{tabular}{|lll|}
\hline COMPOUNDS & $\begin{array}{l}\Delta \text { MD SIMULATIONS (MPA)0.5 } \\
(|\triangle N B V-\triangle C A R R I E R|)\end{array}$ & INTERPRETATION \\
\hline Nebivolol HCL & & Miscible \\
\hline GMO & 1.32 & Miscible \\
\hline VITE TPGS & 3.19 & Immiscible \\
\hline POLOXAMER 407 & 7.49 & \\
\hline
\end{tabular}

Table 3 shows the differences in solubility parameters between NBV and the carriers as predicted by MD simulations. Because of a minimal (1.32 MPa0.5) variation between the two systems, the simulated data suggests that NBV/GMO and NBV/Vit E TPGS are miscible. Because of a minimal (1.32 MPa0.5) variation between the two systems, the simulated data suggests that NBV/GMO and NBV/Vit E TPGS are miscible, on the other hand significant difference be- tween $\Delta \delta$ of NBV/POLOXAMER 407 indicates immiscibility. The miscibility was further verified by DSC.

We also examined the energy contributions to the net solubility parameter from van der Waals (vdW) and electrostatic interactions shown in Table 4. The relative intensities of dispersal and polar forces in the compounds are reflected in these partial solubility characteristics. When the magnitudes of these forces are compared, it is clear that the van der Waals forces ( $v d W$ ) have a significant role in the solubility parameter values for NBV and GMO, VIT E TPGS. As a result, NBV and GMO, Vit-E TPGS have a similar magnitude of energy interactions, which might be due to their miscibility. In Poloxamer 407, the dominance of electrostatic forces might be a reason for their poor or immiscibility with NBV. 
Table 4

Forces Contributing to the MD Solubility

Parameters (MPa0.5)

\begin{tabular}{|c|c|c|}
\hline Compounds & Selectrostatic & $\delta v d W$ \\
\hline NBV & 11.67 & 18.50 \\
\hline GMO & 11.15 & 17.27 \\
\hline VIT E TPGS & 7.15 & 17.24 \\
\hline POLOXAMER 407 & 13.7 & 6.01 \\
\hline
\end{tabular}

\section{Conformation Of Miscibility By Dsc}

Thermo analytical methods such as differential scanning calorimetry can be used to determine the miscibility. In DSC a shift in the drug's melting endotherm, changes in the glass transition temperatures for amorphous systems, or both are indicators of miscibility. The melt endotherm onset temperature (Tm, onset) was employed to assess miscibility in this work. When the chemical potential of the crystalline drug equals the chemical potential of the molten drug, the melting point of a pure drug is reached. If a drug is miscible with a carrier, the drug's chemical potential in the combination is lesser than the pure drug's chemical potential. Exothermic mixing should result in a significant melting point depression but weakly exothermic, athermal and endothermic mixing should result in progressively less melting point depression. The immiscible system had no melting point depression because in presence of a carrier the chemical potential of the drug remains the same.

\subsection{Binary mixtures of Nebivolol HCL, GMO and Vit ETPGS}

The DSC thermogram of Nebivolol HCL is shown in Fig. 6.A. Mixtures of NBV GMO, VIT E TPGS mixtures are shown in Fig. 6.B. Pure Nebivolol $\mathrm{HCl}$ showed a characteristic single sharp endothermic peak at approximately between $210-215 \circ \mathrm{C}$, corresponding to its melting point and crystalline nature. Glycerol mono oleate shows an endothermic melting peak between $40-45^{\circ} \mathrm{C}$ and Vit E TPGS showed an endothermic peak between $40-50^{\circ} \mathrm{C}$. In the range of $30-300^{\circ} \mathrm{C}$, no distinguishable peaks for nebivolol $\mathrm{HCL}$ were found only the broad endothermic peak of Vit E TPGS was observed at approximately $52.94^{\circ} \mathrm{C}$. The Tm melt onset was $48.51{ }^{\circ} \mathrm{C}$.

[Fig. 6.A. and 6.B near here.]

\subsection{Binary mixtures of Nebivolol $\mathrm{HCl}, \mathrm{GMO}$, and Poloxamer 407}

The DSC thermogram of Nebivolol HCL, GMO and Poloxamer 407 mixtures are shown in Fig. 6C. Pure Nebivolol $\mathrm{HCl}$ showed a characteristic single sharp endothermic peak at approximately between 210 $215^{\circ} \mathrm{C}$, corresponding to its melting point and crystalline nature and $\mathrm{GMO}$ showed endothermic melting peak between $40-45^{\circ} \mathrm{C}$ and Poloxamer 407 showed endothermic peak between $50-60^{\circ} \mathrm{C}$. No distinct peaks were observed for Nebivolol $\mathrm{HCl}$ in the range of $30-300^{\circ} \mathrm{C}$. Only the endothermic peak of 
poloxamer 407 was observed at approximately $56.34^{\circ} \mathrm{C}$. Variations in the endothermic peak of Poloxamer 407 can also be linked to the difference in the molecular weight and relative concentration of Polypropylene oxide (PPO) and Polyethylene oxide (PEO) blocks mainly PPO content, directly affect the thermal behavior of pluronic polymers. In addition, in the current study, the shorter PPO chains of Poloxamer 407 (56 units) are used. The melt onset $\mathrm{Tm}$ was at $52.10^{\circ} \mathrm{C}$, decrease in melting point of the system is observed for miscible system (melting point depression) in this case the drug melting peak was absent or decrease in melting point depression occurs this indicates dissolution of the Nebivolol in GMO and Poloxamer 407, hence this confirms miscibility of Poloxamer 407 with Nebivolol and GMO predicted by MD simulations are well correlated with DSC thermograms graph displays that only NBV/VITE TPGS are able to form well-built hydrogen bond interaction to make a stable solid dispersion. Weak hydrogen bond interaction was found for the NBV/POLOXAMER 407 combinations. NBV and Poloxamer 407 are predicted as immiscible which may be due to predominance of electrostatic interaction. Hence this reveals that this combination is not suitable to formulate as a solid dispersion. If we concentrate to design the lipid-based formulations such as SLN, NLC and Cubosomes, the drug must be soluble in lipid but the stabilizer may or may not be miscible within the formulation. NBV/GMO are predicted as miscible and POLOXAMER 407 is predicted as immiscible with Nebivolol hydrochloride. However, DSC observations have confirmed the miscibility of NBV and GMO in Poloxamer 407.

[Fig. 6.C. near here.]

\section{Conclusions}

Molecular dynamics (MD) simulation was carried out to obtain the solubility parameters of drug, lipid and polymers. Solubility parameters derived from simulation are well acknowledging group contribution methods. Solubility parameters derived from the simulation indicates that the combinations of NBV/GMO, NBV/VITE-TPGS, and NBV/GMO/POLOXAMER 407 are doubtlessly to be miscible. Although, $\mathrm{H}$-bond interactions are important for formulations, such as solid dispersion to be stable. Analysis of $\mathrm{H}$ bond interaction graph displays that only NBV/VITE TPGS are able to form well-built hydro- gen bond interaction to make a stable solid dispersion. Weak hydrogen bond interaction was found for the NBV/POLOXAMER 407 combinations. NBV and Poloxamer407 are predicted as immiscible which may be due to predominance of electrostatic interaction. Hence this reveals that this combination is not suitable to formulate as a solid dispersion. If we concentrate to design the lipid-based formulations such as SLN, NLC, Cubosomes the drug must be soluble in lipid but the stabilizer may or may not be miscible within the formulation NBV/GMO are predicted as miscible and POLOXAMER 407 is predicted as immiscible with nebivolol hydrochloride. However, DSC observations have confirmed the miscibility of NBV AND GMO in Poloxamer 407. This finding indicates that nebivolol hydrochloride is miscible with GMO and this combination is further miscible with poloxamer 407 therefore molecular dynamic simulation can be used to understand the molecular level interaction occurs between the molecule. The drug and carrier miscibility were confirmed based on thermal analytical techniques such as DSC (Tg and Tm), but this MD approach is powerful technique to screen the lead excipients during the formulation development stage for the various types of formulation based on solubility parameter prior to thermal analytical techniques. 
Group contribution methods have a drawback that they are based on rules of additivity but MD method had advantage that allows one to investigate systems with functional groups that haven't been parametrised via group contribution methods. Thus, this approach has a potential to minimizing the need of various time consuming thermal analytical techniques and to reduce the cost of formulation development stage.

\section{Declarations}

\section{Acknowledgement}

The authors would like to thank PSG College of Pharmacy for providing all the required facilities for carrying out this work.

\section{Funding}

The authors did not receive support from any organization for the submitted work.

\section{Conflict of interest}

The authors have no competing interests to declare that are relevant to the content of this article.

\section{Availability of data and material}

The data that support the finding of this study are available within the article.

\section{Code availability}

Molecular dynamic simulation using SCHRÖDINGER MATERIALS SCIENCE SUITE Version 21.xin DEMONDV 5.2 .

\section{Author contribution}

All authors contributed to the study conception and design. Material preparation, datacollection, and analysis were performed by [Murugesan Anand Babu], [Murugesan Anand Babu], and [Murugesan Anand Babu and Radhakrishnan Nithya]. The first draft of the manuscript was written by [Murugesan Anand Babu] and all authors commented on previous versions of the manuscript. All authors read and approved the final manuscript.

\section{References}

1. Turpin, E. R., Taresco, V., Al-Hachami, W. A., Booth, J., Treacher, K., Tomasi, S., Alexander, C., Burley, J., Laughton, C. A., \& Garnett, M. C. (2018). In Silico Screening for Solid Dispersions: The Trouble with Solubility Parameters and FH. Molecular Pharmaceutics, 15(10), 4654-4667. https://doi.org/10.1021/acs.molpharmaceut.8b00637 
2. Walden, D. M., Bundey, Y., Jagarapu, A., Antontsev, V., Chakravarty, K., \& Varshney, J. (2021). Molecular Simulation and Statistical Learning Methods toward Predicting Drug-Polymer Amorphous Solid Dispersion Miscibility, Stability, and Formulation Design. Molecules (Basel, Switzerland), 26(1). https://doi.org/10.3390/molecules26010182

3. Gupta, J., Nunes, C., \& Jonnalagadda, S. (2013). A molecular dynamics approach for predicting the glass transition temperature and plasticization effect in amorphous pharmaceuticals. Molecular Pharmaceutics, 10(11), 4136-4145. https://doi.org/10.1021/mp400118v

4. Jadhav, P., Gokarna, V., Deshpande, V., \& Vavia, P. (2020). Bioavailability Enhancement of Olmesartan Medoxomil Using Hot-Melt Extrusion: In-Silico, In-Vitro, and In-Vivo Evaluation. AAPS PharmSciTech, 21(7), 1-17. https://doi.org/10.1208/s12249-020-01780-3

5. Huynh, L., Grant, J., Leroux, J. C., Delmas, P., \& Allen, C. (2008). Predicting the solubility of the anticancer agent docetaxel in small molecule excipients using computational methods. Pharmaceutical Research, 25(1), 147-157. https://doi.org/10.1007/s11095-007-9412-3

6. Mehta, C. H., Narayan, R., \& Nayak, U. Y. (2019). Computational modeling for formulation design. Drug Discovery Today, 24(3), 781-788. https://doi.org/10.1016/j.drudis.2018.11.018

7. Maniruzzaman, M., Pang, J. D., Morgan, D. J., \& Douroumis, D. (2015). Molecular modeling as a predictive tool for the development of solid dispersions. Molecular Pharmaceutics, 12(4), 10401049. https://doi.org/10.1021/mp500510m

8. Das, T., Mehta, C. H., \& Nayak, U. Y. (2020). Multiple approaches for achieving drug solubility: an in silico perspective. Drug Discovery Today, 25(7), 1206-1212.

https://doi.org/10.1016/j.drudis.2020.04.016

9. Hossain, S., Kabedev, A., Parrow, A., Bergström, C. A. S., \& Larsson, P. (2019). Molecular simulation as a computational pharmaceutics tool to predict drug solubility, solubilization processes and partitioning. European Journal of Pharmaceutics and Biopharmaceutics, 137(February), 46-55. https://doi.org/10.1016/j.ejpb.2019.02.007

10. Parekh, V. J., Desai, N. D., Shaikh, M. S., \& Shinde, U. A. (2017). Self nanoemulsifying granules (SNEGs) of meloxicam: preparation, characterization, molecular modeling and evaluation of in vivo anti-inflammatory activity. Drug Development and Industrial Pharmacy, 43(4), 600-610. https://doi.org/10.1080/03639045.2016.1275665

11. Hancock, B. C., York, P., \& Rowe, R. C. (1997). The use of solubility parameters in pharmaceutical dosage form design. International Journal of Pharmaceutics, 148(1), 1-21. https://doi.org/10.1016/S0378-5173(96)04828-4

12. Anderson, B. D. (2018). Predicting Solubility/Miscibility in Amorphous Dispersions: It Is Time to Move Beyond Regular Solution Theories. Journal of Pharmaceutical Sciences, 107(1), 24-33. https://doi.org/10.1016/j.xphs.2017.09.030

13. Birru, W. A., Warren, D. B., Headey, S. J., Benameur, H., Porter, C. J. H., Pouton, C. W., \& Chalmers, D. K. (2017). Computational Models of the Gastrointestinal Environment. 1. The Effect of Digestion on the 
Phase Behavior of Intestinal Fluids. Molecular Pharmaceutics, 14(3), 566-579.

https://doi.org/10.1021/acs.molpharmaceut.6b00888

14. Birru, W. A., Warren, D. B., Han, S., Benameur, H., Porter, C. J. H., Pouton, C. W., \& Chalmers, D. K. (2017). Computational Models of the Gastrointestinal Environment. 2. Phase Behavior and Drug Solubilization Capacity of a Type I Lipid-Based Drug Formulation after Digestion. Molecular Pharmaceutics, 14(3), 580-592. https://doi.org/10.1021/acs.molpharmaceut.6b00887

15. Nithya, R., Jerold, P., \& Siram, K. (2018). Cubosomes of dapsone enhanced permeation across the skin. Journal of Drug Delivery Science and Technology, 48, 75-81.

https://doi.org/10.1016/j.jddst.2018.09.002

16. Shah, M., \& Agrawal, Y. (2013). High throughput screening: an in silico solubility parameter approach for lipids and solvents in SLN preparations. Pharmaceutical Development and Technology, 18(3), 582-590. https://doi.org/10.3109/10837450.2011.635150

17. Makoni, P. A., Ranchhod, J., WaKasongo, K., Khamanga, S. M., \& Walker, R. B. (2020). The use of quantitative analysis and Hansen solubility parameter predictions for the selection of excipients for lipid nanocarriers to be loaded with water soluble and insoluble compounds. Saudi Pharmaceutical Journal, 28(3), 308-315. https://doi.org/10.1016/j.jsps.2020.01.010

18. Lee, Y. C., Dalton, C., Regler, B., \& Harris, D. (2018). Drug solubility in fatty acids as a formulation design approach for lipid-based formulations: a technical note. Drug Development and Industrial Pharmacy, 44(9), 1551-1556. https://doi.org/10.1080/03639045.2018.1483395

19. Maus, M., Wagner, K. G., Kornherr, A., \& Zifferer, G. (2008). Molecular dynamics simulations for drug dosage form development: thermal and solubility characteristics for hot-melt extrusion. Molecular Simulation, 34(10-15), 1197-1207.

20. Bennett-Lenane, H., O’Shea, J. P., O’Driscoll, C. M., \& Griffin, B. T. (2020). A Retrospective Biopharmaceutical Analysis of $>800$ Approved Oral Drug Products: Are Drug Properties of Solid Dispersions and Lipid-Based Formulations Distinctive? Journal of Pharmaceutical Sciences, 109(11), 3248-3261. https://doi.org/10.1016/j.xphs.2020.08.008

21. Harder, E., Damm, W., Maple, J., Wu, C., Reboul, M., Xiang, J. Y., Wang, L., Lupyan, D., Dahlgren, M. K., Knight, J. L., Kaus, J. W., Cerutti, D. S., Krilov, G., Jorgensen, W. L., Abel, R., \& Friesner, R. A. (2016). OPLS3: A Force Field Providing Broad Coverage of Drug-like Small Molecules and Proteins. Journal of Chemical Theory and Computation, 12(1), 281-296. https://doi.org/10.1021/acs.jctc.5b0086

22. Van Krevelen, D. W., \& Te Nijenhuis, K. (2009). Cohesive Properties and Solubility. In Properties of Polymers: Vol. i. https://doi.org/10.1016/b978-0-08-054819-7.00007-8

\section{Figures}


A)GMO
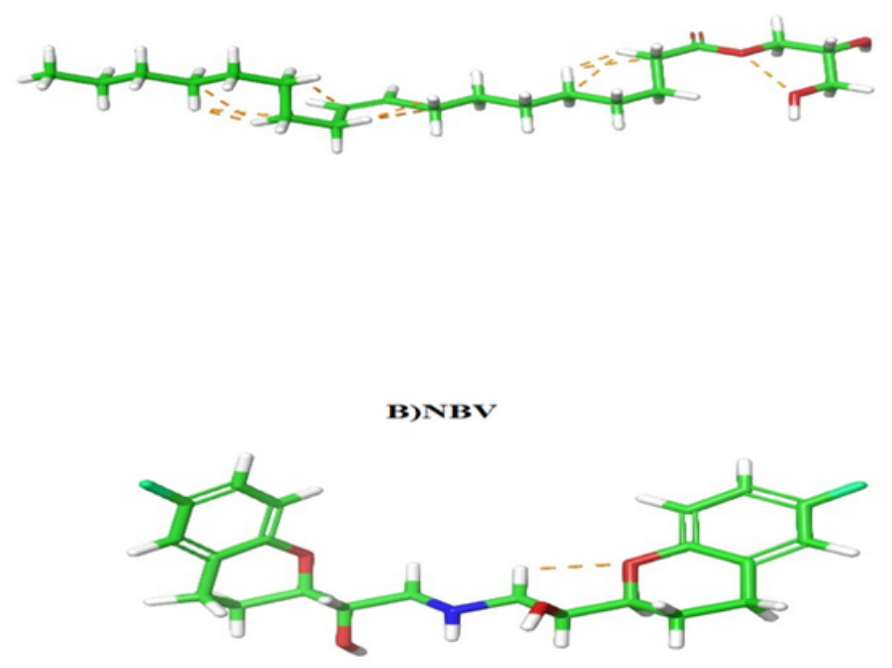

C)VIT-E TPGS
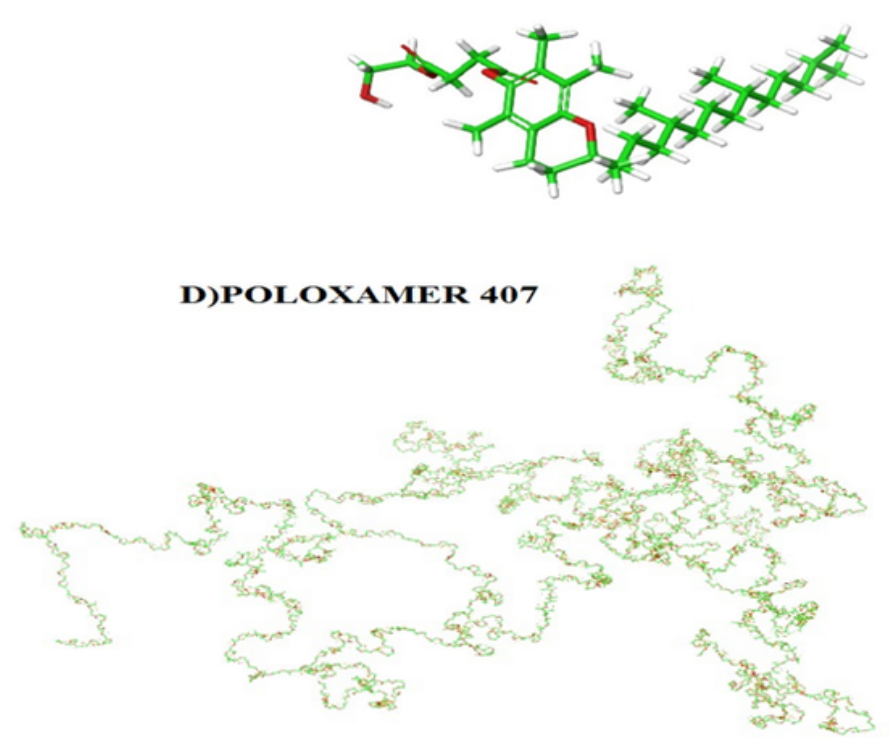

Figure 1

STRUCTURES OBTAINED FROM PUB CHEM a) GLYCEROL MONOOLEATE b) NEBIVOLOL HCL C)Vit-E TPGS D) POLOXAMER 407 


\section{A)VIT-E TPGS}
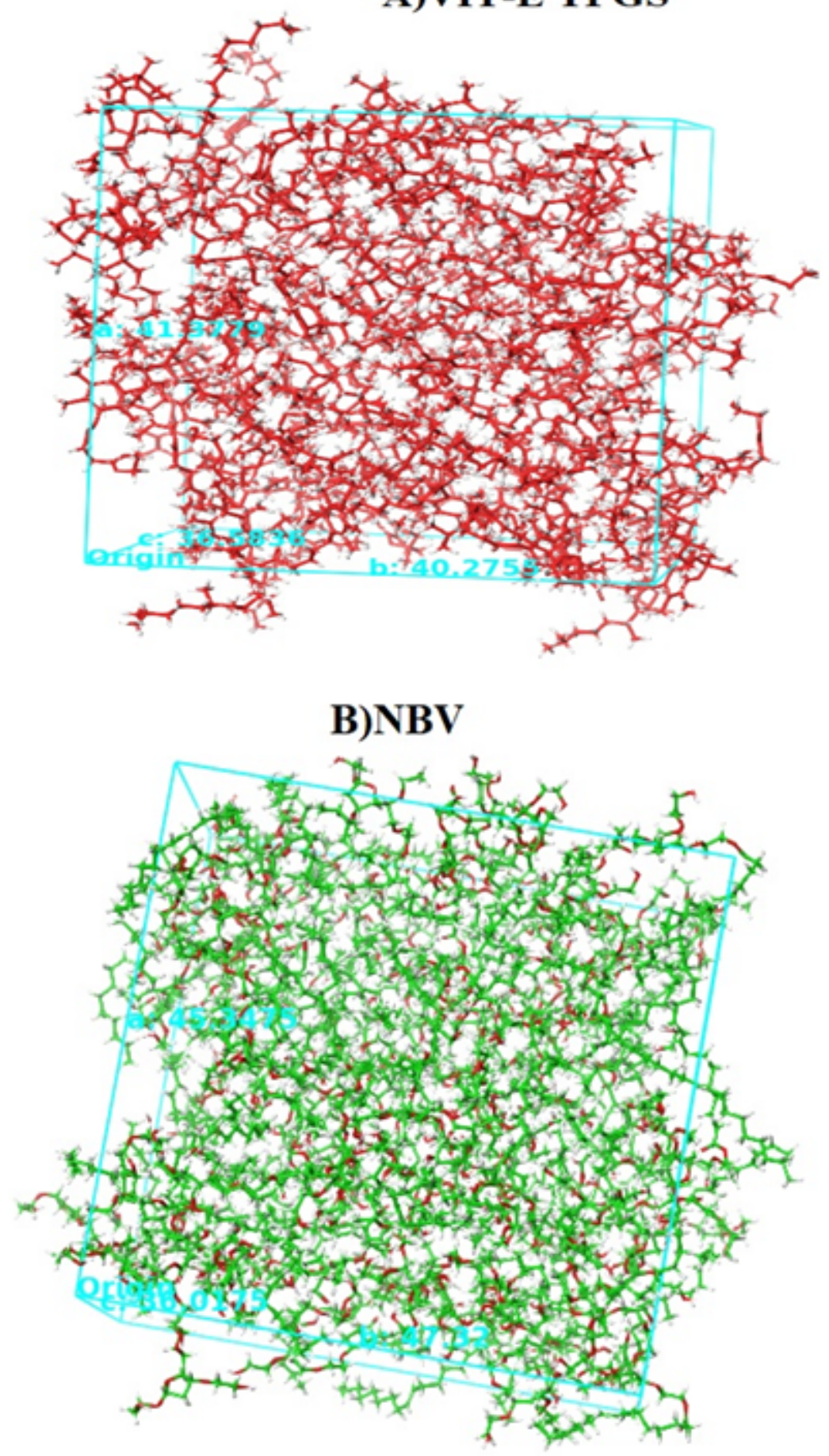

Figure 2

DENSITY MINIMIZED AMORPHOUS CUBIC CELLS A) VIT- E TPGS B) NBV. 


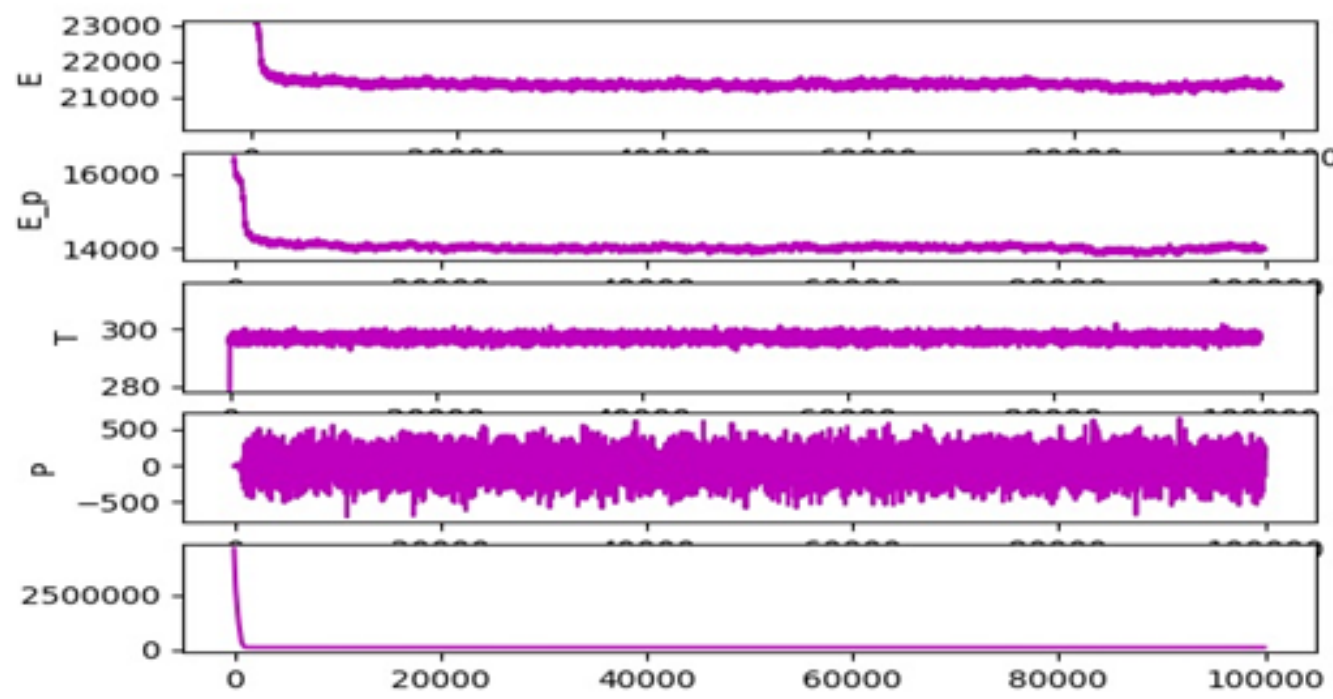

Figure 3

POTENTIAL ENERGY EVALUATION PROFILE

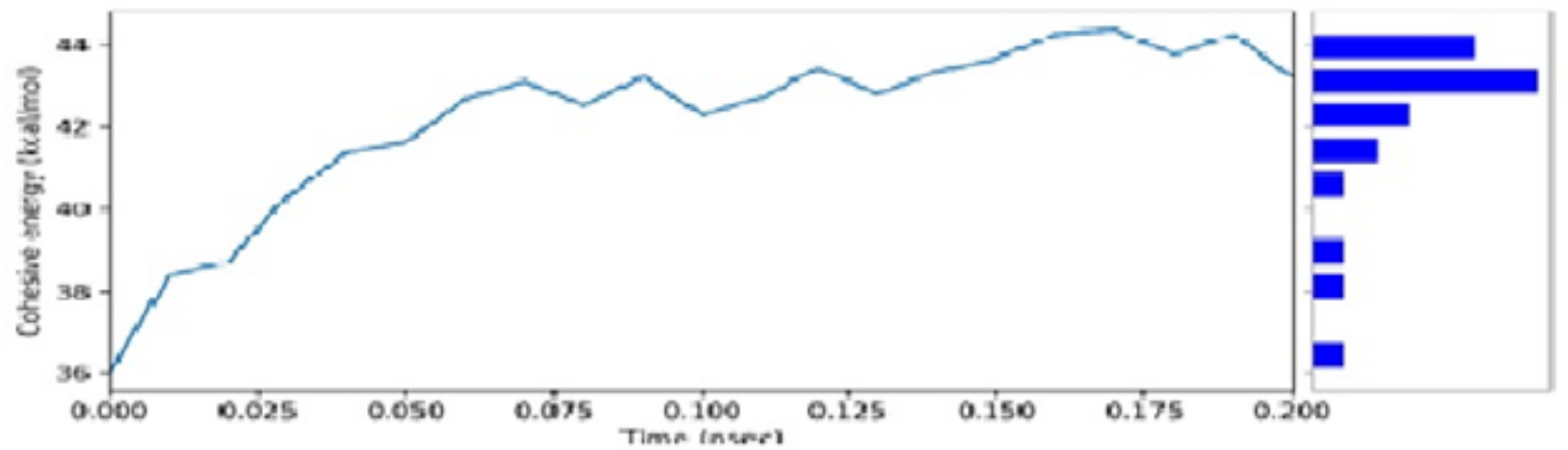

Figure 4

COHESIVE ENERGY DENSITY OF VIT-E TPGS 


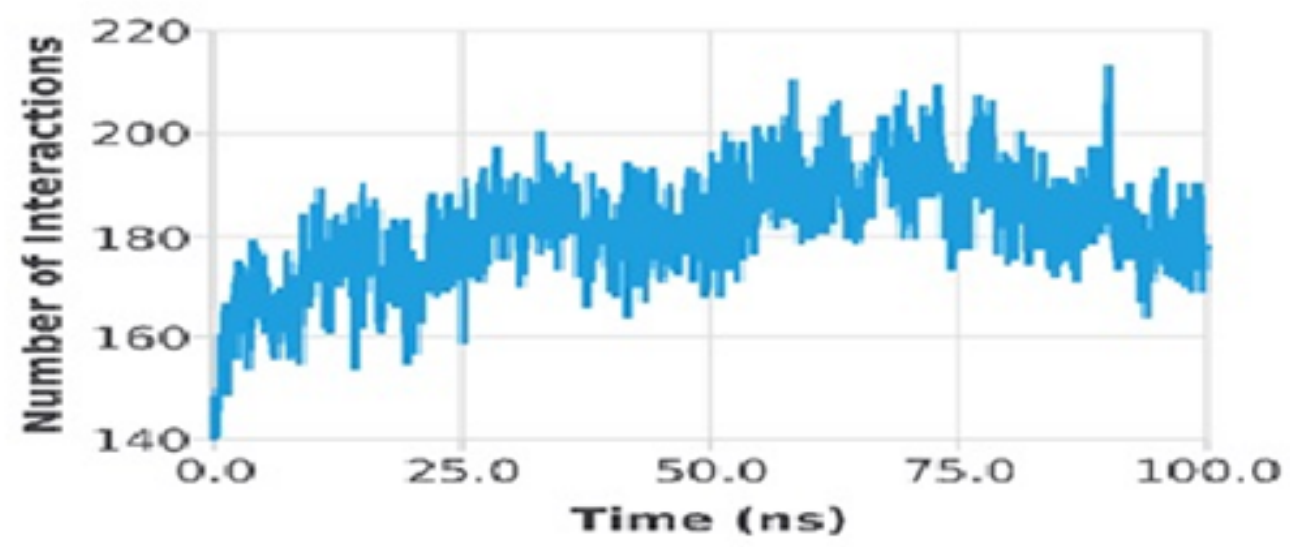

\section{A)NBV}

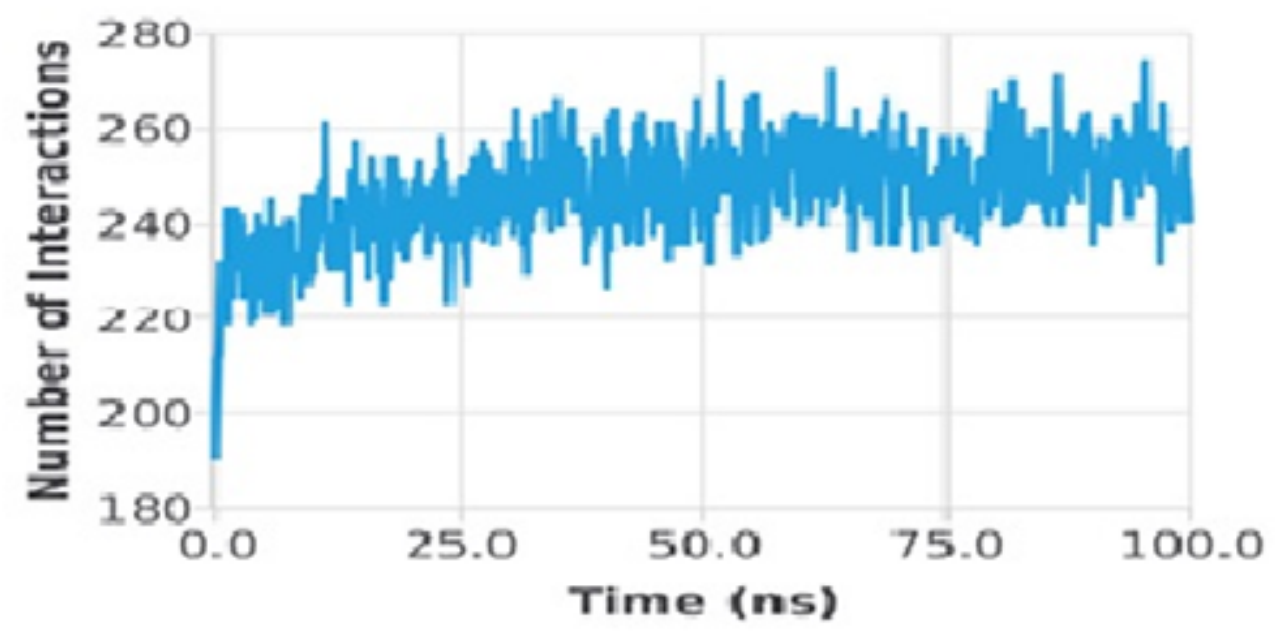

\section{B)VIT-E TPGS}

Figure 5

H-BOND INTERACTION GRAPH A) NBV B) VIT E TPGS 


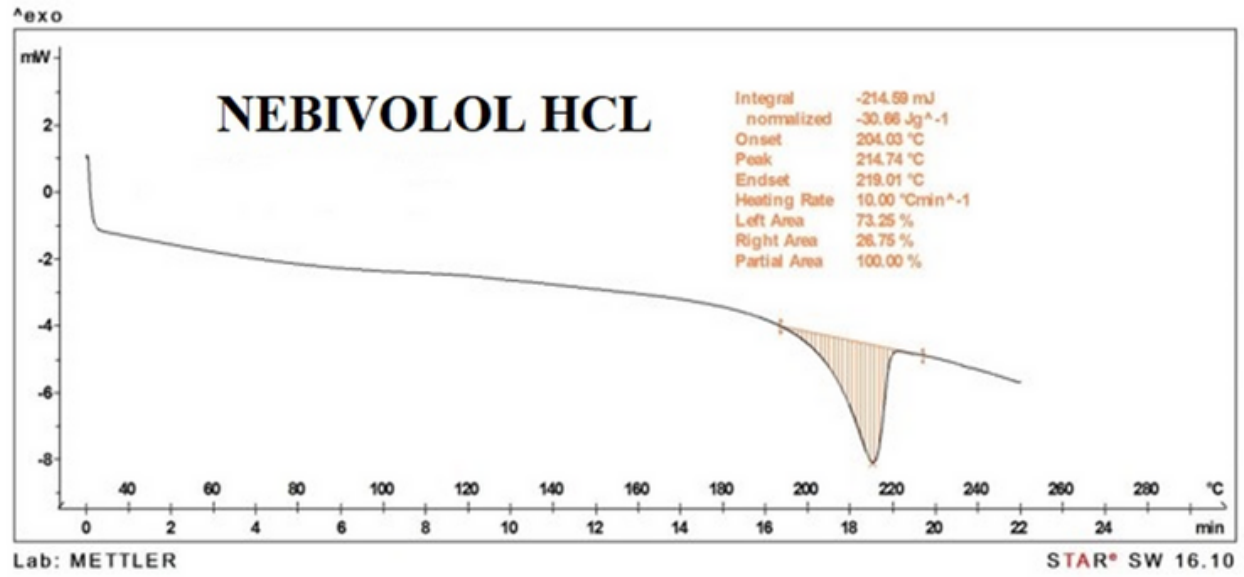

A

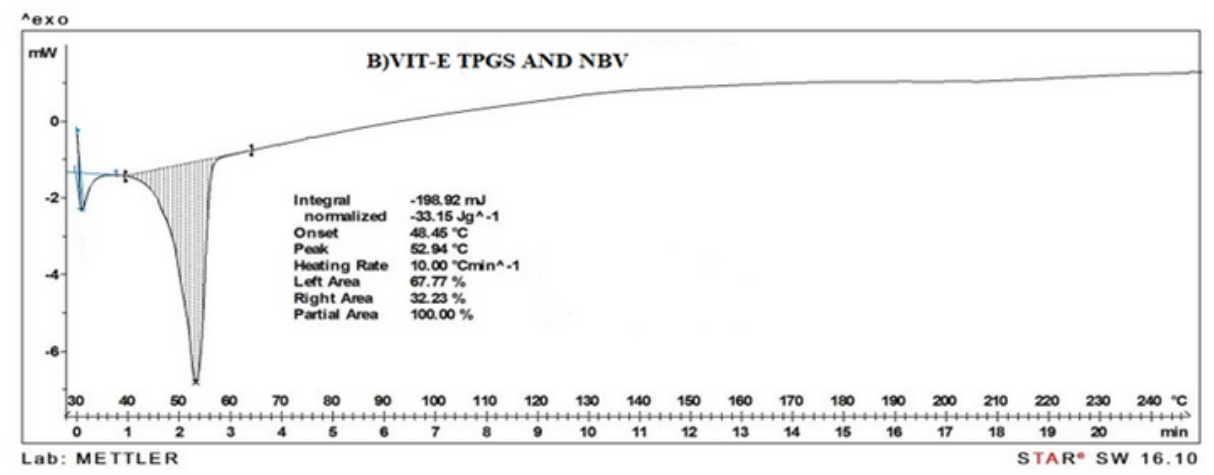

B)

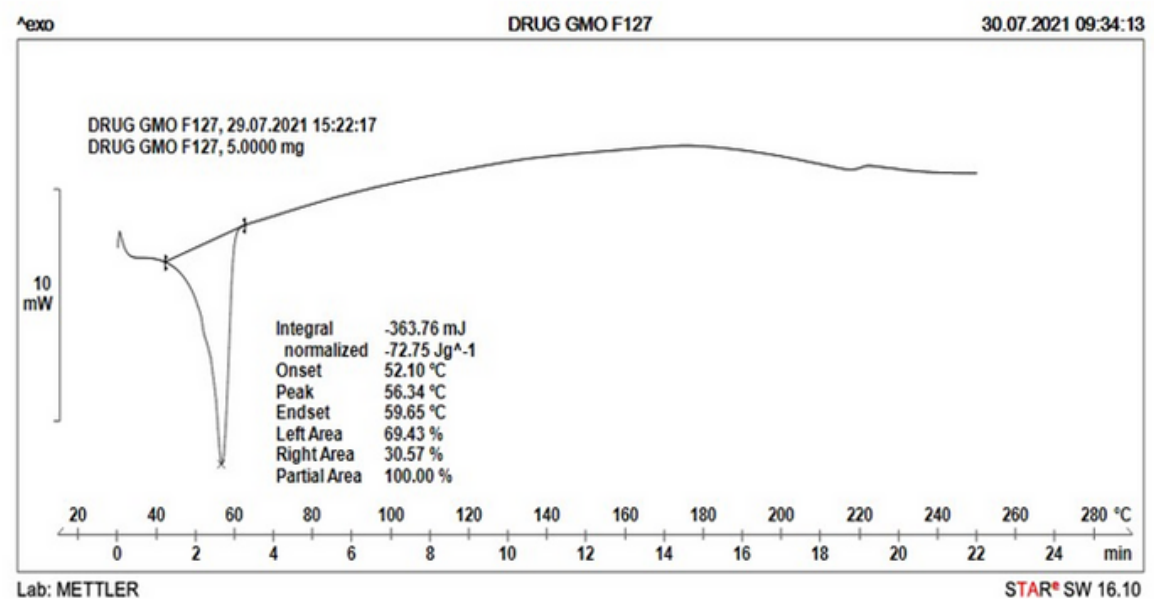

C)

Figure 6

A) DSC THERMOGRAMS OF PURE NBV B) VIT-E TPGS AND NBV C) POLOXAMER 407 AND NBV. 